\title{
The effect of cow's milk consumption on cardiometabolic health in women of childbearing age
}

\author{
A.J. Yeates, N. Gilmartin, S.M. O’Kane, L.K. Pourshahidi, M.S. Mulhern and J.J. Strain \\ Northern Ireland Centre for Food and Health (NICHE), Ulster University, Coleraine, BT52 1SA, UK
}

Cow's milk is a uniquely rich source of high quality protein, vitamins and minerals including riboflavin, vitamin B12, calcium, phosphorus and iodine $e^{(1)}$. It contains variable amounts of fat, comprised predominantly of saturated fatty acids (SFA). This may partly explain the common consumer perception that milk is a high fat food and also why milk consumption rates have declined in recent years, particularly among young females ${ }^{(2)}$. Yet observational evidence suggests a neutral or potentially beneficial association between milk consumption and markers of cardiometabolic health ${ }^{(3-4)}$. The aim of this study was to investigate the impact of increased cow's milk consumption on measures of cardiometabolic health (specifically serum lipids, plasma glucose and anthropometry) in women of childbearing age.

Analysis was carried out on samples and data collected from the 12-week Selenium and Iodine in Milk Intervention (SIMI) study which recruited $n 78$ healthy women (18-45 years). Participants were habitually low consumers of milk $(<250 \mathrm{mls} / \mathrm{d})$ and were randomly assigned into control (no additional milk) or intervention (additional 430mls/d) groups. At 0, 6 and 12 weeks, fasting blood samples were collected from which serum lipid profiles (total cholesterol, HDL and triglycerides) and plasma glucose were analysed (ILab Clinical Analyser, Cheshire). LDL concentrations were calculated using the Friedwald formula. Waist:hip ratio, BMI (kg/m²) and blood pressure $(\mathrm{mmHg})$ were obtained at each time point. Repeated measures ANOVA or Mann-Whitney U and Wilcoxon signed rank tests were conducted according to distribution of data (SPSS v.22, Chersey). The present study was conducted according to the guidelines in the Declaration of Helsinki, and all procedures were approved by the Ulster University Research Ethics Committee.

No significant differences were observed between groups for changes in waist:hip ratio, BMI, plasma glucose, total cholesterol, HDL cholesterol or triglyceride concentrations as a result of the intervention. A significant time*treatment effect was observed for diastolic blood pressure (DBP) which increased in the control compared to the intervention group $(P=0 \cdot 03)$. Fig. 1 shows a significant decrease in LDL cholesterol over time in the intervention group compared to control group $(P<0 \cdot 05)$. The time*treatment interaction was not significant.

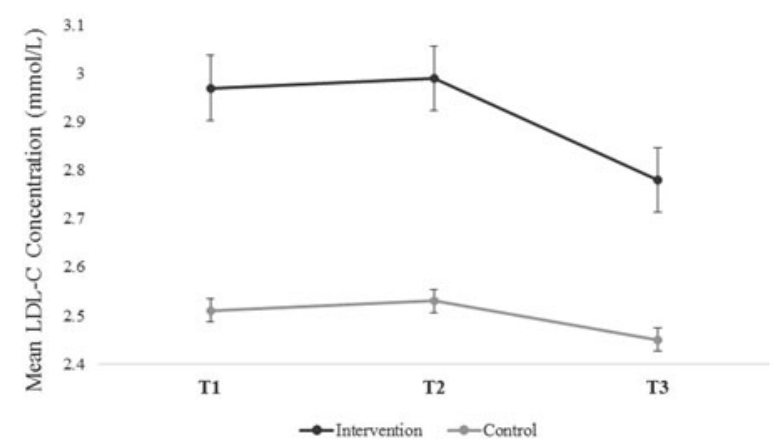

Fig.1. Serum LDL concentrations (mmol/L).

Overall, increased milk consumption did not negatively impact any marker of cardiometabolic health. Indeed, the notable decrease in LDL cholesterol concentrations within the intervention group suggests a beneficial effect of increased milk consumption which warrants further investigation.

1. Food and Agriculture Organisation (2013). Milk and dairy products in human nutrition. http://www.fao.org/docrep/018/i3396e/i3396e.pdf.

2. Department for Health (2014). National Diet and Nutrition Survey. Results from Years 1,2,3 and 4 (combined) of the Rolling Programme (2008/ 2009-2011/12).

3. Ralston R, Lee J, Truby H et al. (2012) J Hum Hypertens 26, 3-13.

4. Mozaffarian D, Hao T, Rimm EB et al. (2011) New Engl J Med 364, 2392-2402. 